\title{
WORKSHOP
}

\section{ON CHANGING THE PARAMETER OF EXPONENTIAL SMOOTHING IN EXPERIENCE RATING}

\author{
BY HEIKKI BONSDORFF \\ Pohjola Insurance Company Ltd., \\ Helsinki, Finland
}

\begin{abstract}
We consider exponential smoothing $Y_{n}=\alpha X_{n}+(1-\alpha) Y_{n-1}, 0<\alpha<1$, in experience rating. Here the premium $Y_{n}$ is determined by the policy's own claims history $\left(X_{n}\right)$. In order to uniformize the fluctuation of premiums, it is appropriate to use a bigger $\alpha$ for the big policies than for the small ones. When the size of the policy changes with time, a need arises to change $\alpha$ correspondingly. It has recently been shown that changing based on the size of the premiums $Y_{n}$ may lead to too low a tariff level. This result is presented here and illustrated by means of simulation. Further, some general results are given how the changing can be made without a decline in the tariff level. The results are applied to a tariff system in which the linking of the smoothing parameter to the size of the policy is particularly motivated.
\end{abstract}

\section{KEYWORDS}

Exponential smoothing; adaptive smoothing; experience rating; Markov chains; big claims; workers' compensation insurance.

\section{INTRODUCTION}

In this paper we consider certain questions related to applications of exponential smoothing to premium rating. Especially, we deal with problems concerning changing the smoothing parameter.

Let $\left(X_{n}, n \geq 1\right)$ be a sequence of random variables. Exponential smoothing $\left(Y_{n}\right)$ of the sequence $\left(X_{n}\right)$ is defined by

$$
Y_{n}=\alpha X_{n}+(1-\alpha) Y_{n-1},
$$

where $\alpha$ is a constant, $0<\alpha<1$.

Exponential smoothing is often used for defining the premiums $Y_{n}$ on the basis of the policy's own claims history $\left(X_{n}\right)$ (cf. e.g. [5] and [6]). If the random variables $X_{n}$ have a common expectation, then the premiums $Y_{n}$ are (asymptotically) unbiased. With suitable additional assumptions exponential smoothing leads to a strong correspondence between cumulative premiums and claims of an individual policy. E.g. if the random variables $X_{n}$ are uncorrelated and have a common variance, then the variance of the cumulated difference of claims and premiums remains bounded (cf. [2], p. 288). 
See e.g. [3], [4] and [7] for the connections of exponential smoothing with credibility theory.

Throughout this paper, $X_{n}$ is interpreted as the total claim amount at year $n$, or some modification of it, of a given policy.

We consider policies consisting of distinct similar risks the number of which essentially varies from one policy to another. An example is served by the workers' compensation insurance, where the total risk consists of the risks related to the workers of the policyholder.

The bigger the smoothing parameter $\alpha$ is, the more closely the premiums follow the fluctuation of the claims history. For policies like the above, the fluctuation of the claims history is relatively bigger for small policies than for big ones, where the size of the policy is measured by the (risk) premium. As a consequence, if the same smoothing constant $\alpha$ were used for all policies, also the premiums of the small policies would fluctuate more than those of the big ones. Therefore, it is natural to use a smaller $\alpha$ for the small policies than for the big ones, i.e. to define $\alpha$ as a monotonically increasing function of the premium.

When the size of the policy changes with time, a need arises to vary $\alpha$, as above, monotonically increasingly with respect to the premium. In a recent paper [1], it is shown, in case $X_{n}$ i.i.d., that this kind of varying procedure leads to too low a premium level, cf. (1.3) below. This result is based on the theory of Markov chains on a general state space.

The above paper considers the smoothing procedure

$$
Z_{n}=\beta\left(Z_{n-1}\right) X_{n}+\left(1-\beta\left(Z_{n-1}\right)\right) Z_{n-1},
$$

where $\beta$ is a monotonically increasing function $[0, \infty) \rightarrow[c, d], 0<c<d<1$. It follows from Theorem 4 of [1] that $E Y_{n}, E Z_{n}$ converge with a geometric convergence rate to their limits $E_{Y}, E_{Z}$ and that

$$
E_{Z}<E_{Y}=E X_{n} \text {. }
$$

This result is based on Theorem 3 of [1] which states that $\left(Y_{n}\right)$ and $\left(Z_{n}\right)$ are geometrically ergodic Markov chains (see [1] for definition). The limits $E_{Y}, E_{Z}$ are the means of the invariant probability distributions of the Markov chains concerned. These results are presented in Section 2 in greater detail.

We are concerned in this paper with a reduced model whose stability properties can be studied. Our purpose is to call attention to the phenomenon where the asymptotic value is below the value expected. We believe that this kind of phenomenon may occur also in more general related models.

In Section 3 the result (1.3) is illustrated by means of simulation.

Sections 4 and 5 deal with how the changing of the smoothing parameter, in certain cases, can be controlled without the above-mentioned descending of the tariff level. In Section 4 we present some general results and in Section 5 these are applied to a tariff system, in which linking the smoothing parameter to the size of the policy is motivated, not only because of the uniformizing of the fluctuation of premiums as such, but also because of the equitability of the policies. 


\section{VARYING THE SMOOTHING PARAMETER ON THE BASIS} OF THE PREMIUMS

In the following we present results related to varying the smoothing parameter as a monotonically increasing function of the premium. These results are from [1]. For unexplained concepts the reader is referred to that paper.

Let $X_{n}, n \geq 1$ be i.i.d. random variables taking non-negative values. Assume $E X_{n}<\infty$ and that $X_{n}$ has a continuous density function $g>0$ on $(0, \infty)$ (we allow $\left.P\left(X_{n}=0\right)>0\right)$.

Let $Y_{o}, Z_{o}$ be random variables taking non-negative values and let iteratively for $n \geq 1$

$$
\begin{aligned}
& Y_{n}=\alpha X_{n}+(1-\alpha) Y_{n-1}, \\
& Z_{n}=\beta\left(Z_{n-1}\right) X_{n}+\left(1-\beta\left(Z_{n-1}\right)\right) Z_{n-1},
\end{aligned}
$$

where $\alpha \in(0,1)$ and $\beta:[0, \infty) \rightarrow[c, d]$ is monotonically increasing, $0<c<d<1$.

Here $\left(Y_{n}\right)$ and $\left(Z_{n}\right)$ are interpreted as (alternative) sequences of premiums determined by the annual claims amounts $\left(X_{n}\right)$ of a given policy.

With the above assumptions we have the following results.

Theorem 2.1. The sequences $\left(Y_{n}\right)$ and $\left(Z_{n}\right)$ are geometrically ergodic Markov chains (with state space $S=[0, \infty)$ ).

It follows from Theorem 2.1 that the chains $\left(Y_{n}\right),\left(Z_{n}\right)$ possess unique invariant probability measures $\pi_{Y}, \pi_{Z}$ with corresponding means $E_{Y}, E_{Z}$, respectively.

\section{Theorem 2.2.}

(i) There exist functions $C_{Y}, C_{Z}<\infty$ and constants $\rho_{Y}, \rho_{Z} \in(0,1)$ such that

$$
\begin{aligned}
& \left|E Y_{n}-E_{Y}\right| \leq C_{Y}\left(y_{o}\right) \rho_{Y}^{n}, \\
& \left|E Z_{n}-E_{Z}\right| \leq C_{Z}\left(z_{o}\right) \rho_{Z}^{n}
\end{aligned}
$$

for all $n$, for all initial states $y_{o}$ and for almost all initial states $z_{o}$ (with respect to the Lebesgue measure).

(ii) $E_{Z}<E_{Y}=E X_{n}$.

If the random variables $X_{n}$ and the initial values $Y_{o}, Z_{o}$ take their values in a finite interval $[0, M]$, the above result can be sharpened. In this case, under the same assumptions as above (in particular, assuming that $g$ is continuous and positive on $(0, M)$ and allowing $\left.P\left(X_{n}=0\right)>0, P\left(X_{n}=M\right)>0\right)$ the result (i) of Theorem 2.2 can be replaced by the following one:

(i') There exist constants $C_{Y}, C_{Z}>0$ and $\rho_{Y}, \rho_{Z} \in(0,1)$ such that

$$
\left|E Y_{n}-E_{Y}\right| \leq C_{Y} \rho_{Y}^{n},
$$




$$
\left|E Z_{n}-E_{Z}\right| \leq C_{Z} \rho_{Z}^{n}
$$

for all $n$ and for all initial states $y_{o}, z_{o} \in[0, M]$.

In this case the chains $\left(Y_{n}\right)$ and $\left(Z_{n}\right)$ are uniformly ergodic on the state space $S=[0, M]$.

\section{A SIMULATION EXAMPLE}

The purpose of the simulation is, in a simple case, to give a picture of how much smaller $E_{Z}$ is than $E_{Y}$, cf. (1.3). One can broaden the picture by examining other cases and using also numerical methods in addition to simulation.

The computing was carried out by an IBM Personal Computer XT. The simulation was arranged as follows. The variables $X_{n}, n \geq 1$ were taken independent and uniformly distributed over $[0,1]$. We fixed

$$
\begin{aligned}
& Y_{o}=Z_{o}=0.5, \\
& \alpha=0.5 .
\end{aligned}
$$

As $\beta$ we used

$$
\beta(z)=0.2+0.6 z, 0 \leq z \leq 1 \text { (thus } c=0.2 \text { and } d=0.8 \text { ). }
$$

Using the constants and the function $\beta$ above, we calculated $Y_{n}, Z_{n}$, $n=10,50$.

We ran the simulation 2000 times and calculated the sample means and $99 \%$-confidence intervals (using normal approximation) for the variables $Y_{n}$, $Z_{n}$ and for completeness also for $X_{n}, n=10,50$.

These quantities are presented in the table below. One can see that $Z$ is considerably smaller than $Y$ already at time $n=10$.

TABLE 3.1

\begin{tabular}{lccc}
\hline & Sample mean & Confidence interval \\
\hline$n=10$ & & & \\
$X$ & 0.5067 & 0.4902 & 0.5232 \\
$Y$ & 0.5035 & 0.4939 & 0.5131 \\
$Z$ & 0.4696 & 0.4601 & 0.4791 \\
$n=50$ & & & \\
$X$ & & & 0.5243 \\
$Y$ & 0.5077 & 0.4910 & 0.5138 \\
$Z$ & 0.5042 & 0.4947 & 0.4783 \\
\hline
\end{tabular}

The sample mean and its confidence interval were calculated for $Z_{10}$ as follows, and similarly for the other variables: 
sample mean $\bar{Z}_{10}=\frac{1}{2000} \sum_{k=1}^{2000} Z_{10, k}$

where $Z_{10, k}$ is the value of $Z_{10}$ at the $k^{\prime}$ th run,

$$
\text { confidence interval }=\bar{Z}_{10} \pm 2.58 \frac{S_{Z, 10}}{\sqrt{2000}}
$$

where $S_{Z, 10}=\left[\frac{1}{2000} \sum_{k=1}^{2000}\left(Z_{10, k}-\bar{Z}_{10}\right)^{2}\right]^{1 / 2}$.

\section{VARYING THE SMOOTHING PARAMETER WITHOUT CORRELATION WITH THE CLAIMS HISTORY}

In the following, we consider certain conditions under which the varying of the smoothing parameter can be done without a decline in the premium level (cf. Theorem 2.2). First, we show that this works if the varying procedure is such that the smoothing parameters do not correlate with the claims history, see Theorem 4.1 below. We then apply Theorem 4.1 to a case in which the premium $P_{n}$ is presented in the form

$$
P_{n}=V_{n} p_{n},
$$

where $V$ is a volume measure of the policy and $p$ a measure of risk per volume unit derived from the claims history of the policy. The volume measure $V$ can be e.g. the number of similar subrisks. It turns out that, under certain assumptions, the varying procedure of the smoothing parameter can be based on $V$ so that the premiums are unbiased, see Corollary 4.2 below.

Theorem 4.1. Let $\left(X_{n}\right)$ be a sequence of random variables taking non-negative values, with $E X_{n}=a<\infty$ for all $n$. Let $Z_{o}>0$ and let

$$
Z_{n}=\alpha_{n} X_{n}+\left(1-\alpha_{n}\right) Z_{n-1}, \quad \text { for } n \geq 1,
$$

where the $\alpha_{n}$ 's are random variables satisfying the conditions

$$
0<c \leq \alpha_{n} \leq d<1
$$

and

$$
\begin{aligned}
& E\left(\left(1-\alpha_{n}\right)\left(1-\alpha_{n-1}\right) \cdots\left(1-\alpha_{k+1}\right) \alpha_{k} X_{k}\right)= \\
& E\left(\left(1-\alpha_{n}\right)\left(1-\alpha_{n-1}\right) \cdots\left(1-\alpha_{k+1}\right) \alpha_{k}\right) E X_{k}
\end{aligned}
$$

for all $k, n ; k \leq n$.

Then

$$
\left|E Z_{n}-a\right| \leq\left|Z_{o}-a\right|(1-c)^{n} \text { for all } n \text {. }
$$


Proof. We have

$$
\begin{aligned}
Z_{n}=\alpha_{n} & X_{n}+\left(1-\alpha_{n}\right) \alpha_{n-1} X_{n-1}+\left(1-\alpha_{n}\right)\left(1-\alpha_{n-1}\right) \alpha_{n-2} X_{n-2} \\
& +\cdots+\left(1-\alpha_{n}\right)\left(1-\alpha_{n-1}\right) \cdots\left(1-\alpha_{2}\right) \alpha_{1} X_{1} \\
& +\left(1-\alpha_{n}\right)\left(1-\alpha_{n-1}\right) \cdots\left(1-\alpha_{1}\right)\left[a+\left(Z_{o}-a\right)\right] .
\end{aligned}
$$

Using (4.3) we get

$$
\begin{gathered}
E Z_{n}=a E\left(\alpha_{n}+\left(1-\alpha_{n}\right) \alpha_{n-1}+\cdots+\left(1-\alpha_{n}\right)\left(1-\alpha_{n-1}\right) \cdots\left(1-\alpha_{2}\right) \alpha_{1}\right. \\
\left.+\left(1-\alpha_{n}\right)\left(1-\alpha_{n-1}\right) \cdots\left(1-\alpha_{1}\right)\right) \\
+\left(Z_{o}-a\right) E\left(\left(1-\alpha_{n}\right)\left(1-\alpha_{n-1}\right) \cdots\left(1-\alpha_{1}\right)\right) .
\end{gathered}
$$

It is easy to see that

$$
\begin{gathered}
\alpha_{n}+\left(1-\alpha_{n}\right) \alpha_{n-1}+\cdots+\left(1-\alpha_{n}\right)\left(1-\alpha_{n-1}\right) \cdots\left(1-\alpha_{2}\right) \alpha_{1} \\
+\left(1-\alpha_{n}\right)\left(1-\alpha_{n-1}\right) \cdots\left(1-\alpha_{1}\right)=1 \text { for all } n
\end{gathered}
$$

Thus

$$
E Z_{n}-a=\left(Z_{o}-a\right) E\left(\left(1-\alpha_{n}\right)\left(1-\alpha_{n-1}\right) \cdots\left(1-\alpha_{1}\right)\right) .
$$

Since $\left(1-\alpha_{k}\right) \leq 1-c$ for all $k$, the assertion follows from (4.5).

Note that condition (4.3) does not hold true for $\left(Z_{n}\right)$ in case of Theorems 2.1 and 2.2. We apply Theorem 4.1 to a claims process satisfying the following assumption.

Assumption (4.6). Let a risk at year $n$ consist of $N_{n}$ subrisks $\eta_{n, 1}, \ldots, \eta_{n, N_{n}}$ for which

$$
E_{\eta_{n, k}}=a<\infty \text { for all } n, k \text {. }
$$

The numbers of subrisks $N_{n}$ are here random variables, $E N_{n}<\infty$ for all $n$. We assume that the variables $\eta_{n, k}$ are independent of the process $\left(N_{n}\right)$. (We allow mutual correlation of the variables $\eta_{n, k}$ as well as that of the variables $N_{n}$.) Denote $\xi_{n}=\sum_{i=1}^{N_{n}} \eta_{n, i}$ and $X_{n}=\xi_{n} / N_{n}$.

Under assumption (4.6) and using the notation above, we have the following corollary of Theorem 4.1 .

Corollary 4.2. Let $\alpha$ be a function of the number of the subrisks $N$ satisfying $0<c \leq \alpha(N) \leq d<1$. Then

(i) relation (4.4) holds true for the process

$$
Z_{n}=\alpha_{n} X_{n}+\left(1-\alpha_{n}\right) Z_{n-1}, n \geq 1,
$$

where $\alpha_{n}=\alpha\left(N_{n-1}\right)$,

(ii) if $P_{n}=Z_{n} N_{n}$, then 


$$
\frac{\left|E P_{n}-E \xi_{n}\right|}{E N_{n}} \leq\left|a-z_{o}\right|(1-c)^{n},
$$

where $z_{o}$ is the initial value of the process (4.7).

\section{Proof.}

(i) Clearly, $E X_{n}=a$ for all $n$. We have to verify condition (4.3). Denote $\left(1-\alpha_{n}\right) \cdots\left(1-\alpha_{k+1}\right) \alpha_{k}=\alpha(k, n)$. Since the variables $\eta_{k, i}$ are independent of the process $\left(N_{n}\right)$, they are independent of $\alpha(k, n)$. Hence

$$
E\left(\alpha(k, n) \eta_{k, i}\right)=E \alpha(k, n) E \eta_{k, i}=a E \alpha(k, n)
$$

for all $k, n, i ; k \leq n, i \leq N_{k}$. Condition (4.3) then follows from (4.9).

(ii) By the independence assumption of (4.6), $E \xi_{n}=a E N_{n}$. By a similar reasoning as in the proof of Theorem 4.1 and in that of item (i) above, we see that $E P_{n}=a E N_{n}+\left(z_{o}-a\right) E\left(\left(1-\alpha_{n}\right) \cdots\left(1-\alpha_{1}\right) N_{n}\right)$. Accordingly, the assertion follows from condition $c \leq \alpha_{n}$.

Consequently, in this case where the varying of the smoothing parameter is based on the volume measure $N_{n}$ instead of the premiums, the premiums keep the right level.

Note that the variables $X_{n}$ in assumption (4.6) need not be independent of the parameters $\alpha_{n}$. If, for instance, $\alpha$ is a monotonically increasing function of the number of the subrisks $N$, then in case $\alpha$ is big (small) then also $N$ is big (small) and $X$ is more (less) concentrated. Note also that the variables $\eta_{n, i}$, $i=1, \ldots, N_{n}$ are allowed to be mutually correlated. This fact has significance in the application presented in Section 5.

Condition $1^{\circ}$ in assumption (4.6) can be weakened. For example, it is easy to see that Collorary 4.2 remains valid if condition $\left(1^{\circ}\right)$ is replaced by the following one :

$\left(2^{\circ}\right)$ The portfolio is composed of classes $C^{j}, j=1, \ldots, m$ such that each class consists of $N_{n}^{j}$ subrisks $\eta_{n, 1}^{j}, \ldots, \eta_{n, N_{n}^{\prime}}^{j}$ for which

$$
E \eta_{n, k}^{j}=a_{j}<\infty \text { for all } n, k,
$$

and that the ratios $r_{j}=N_{n}^{j} / N_{n}$ are constants. (In this case $a=\sum_{j} r_{j} a_{j}$.)

In workers' compensation insurance the subclasses $C^{j}$ can be interpreted as different occupational groups.

\section{A TARIFF SYSTEM IN WORKERS' COMPENSATION INSURANCE}

The following presentation is founded on a Finnish tariff system for workers' compensation insurance. The tariff (in the following FT) is intended for medium-sized and bigger employers and is applied since 1983. The tariff aims 
at a relatively strong correspondence between claims and premiums of individual policies. The premium is mainly determined by smoothing the policy's own claims history. However, in order to decrease the effects of large claims amounts, also a collective part of the premium is charged. 4.1)

For each policy, the premium $P_{n}$ of year $n$ is presented in the form (cf.

$$
P_{n}=V_{n} p_{n} .
$$

Here $V_{n}$ is the payroll of the policyholder at year $n$. The risk per wage monetary unit measure $p_{n}$ will be defined later. Let

$$
X_{n}=\xi_{n} / V_{n},
$$

where $\xi_{n}$ is the policy's total claims amount at year $n$. The purpose of the tariff is to smooth the possibly strongly fluctuating time series $\left(X_{n}\right)$ to a less fluctuating sequence of coefficients $\left(p_{n}\right)$.

We restrict, in this context, the presentation of the application of the tariff to the case where there is not any significant trend in $\left(X_{n}\right)$ and the risk structure of the policy does not essentially change. (Note that the idemnifications are, for the most part, tied to the wage-index and follow this index closely. As a consequence, a trend in $\left(X_{n}\right)$, caused by inflation, can be regarded as negligible.) In case of a trend or structural change the tariff will not be straightforwardly applied. (See e.g. [2] for controlling this type of situations.)

The time series $\left(X_{n}\right)$ is smoothed, first, by taking a moving average

$$
X_{n}^{*}=\sum_{k=1}^{m} c_{k} X_{n-k},
$$

where $c_{i} \geq 0, \Sigma c_{i}=1, m<\infty$. (In FT $c_{1}=0.5, c_{2}=0.3, c_{3}=0.2$.)

Then exponential smoothing is applied to $\left(X_{n}^{*}\right)$ resulting in the sequence $\left(Z_{n}\right)$, defined as follows

$$
\begin{aligned}
& Z_{o}=z_{o}, \\
& Z_{n}=\alpha_{n} X_{n}^{*}+\left(1-\alpha_{n}\right) Z_{n-1}, \quad \text { when } n \geq 1,
\end{aligned}
$$

where $z_{o}$ is an initial value and $\alpha_{n}$ 's are the smoothing parameters. (A motivation for the double smoothing is that the change in the premiums, caused by a large claims amount, has a flatter shape than what would be the case if only exponential smoothing were applied.) We will revert to the definition of $\alpha_{n}$ 's later. The varying procedure of the smoothing parameters will be such that $\left(Z_{n}\right)$ can be considered to represent a correct tariff level (cf. Section 4 and the discussion at the end of this section).

The smoothing procedure (5.3) is such that, as an effect of a big claims amount in some year, $\left(Z_{n}\right)$ can increase remarkably. Consequently, $\left(Z_{n}\right)$ is not suitable for $\left(p_{n}\right)$, since an even development of the premiums is desirable from the policyholder's point of view. For this reason, the increase of the premiums is reduced by introducing the variables $q_{n}$, defined as follows 


$$
\begin{aligned}
& q_{o}=z_{o}, \\
& q_{n}=\min \left(Z_{n}, h q_{n-1}\right), \quad \text { for } n \geq 1,
\end{aligned}
$$

where $h>1$ is a coefficient limiting the increase of the process $\left(q_{n}\right)$. (In FT $h=1.5$.) See Figure 5.1.

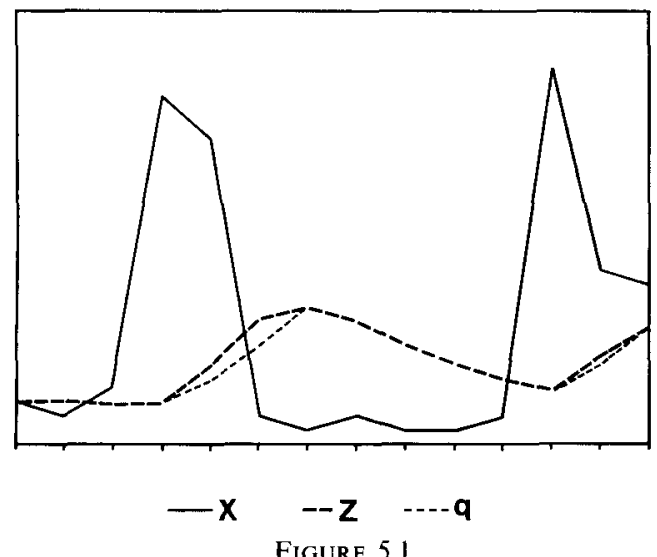

Figure 5.1 .

Clearly, $q_{n} \leq Z_{n}$. As mentioned above, $\left(Z_{n}\right)$ can be considered to represent a correct tariff level. Due to this, all policies are charged a collective part of the premium corresponding to the expected difference $Z_{n}-q_{n}$ over the whole insurance portfolio. The coefficient $p_{n}$ (cf. 5.1) is defined as follows

$$
p_{n}=\left(1+r_{n}\right) q_{n},
$$

where the coefficient $r_{n}$ is common for all policies and $r_{n} q_{n} V_{n}$ is the collective part of the premium of the individual policy in question. (In FT $r_{n}$ has been 0.02 .)

We turn to the definition of the smoothing parameter $\alpha$. Since the policies consist of several similar risks the number of risks varying from one policy to another, the fluctuation of the claims process is steeper for small policies than for bigger ones. This steeper fluctuation would be transmitted also to $\left(Z_{n}\right)$ if the same smoothing constant were applied for all policies. In this case $\left(q_{n}\right)$ of the small policies would differ more from the correct tariff level $\left(Z_{n}\right)$ than $\left(q_{n}\right)$ of the big policies. As a consequence, the small policies would line their pockets at expence of the big ones. For this reason, and also for harmonizing the fluctuation of the premiums, it is reasonable to use a smaller $\alpha$-coefficient for small policies than for big ones.

In the following, we first consider the choosing of $\alpha$ when a policy starts in the tariff system considered. The insurance portfolio is divided, on the basis of the size of the premiums, into classes $C_{i}, i=1, \ldots, l$, where the classes are in increasing order according to the premium size. The limits of the classes are adjusted yearly on the basis of the wage-index. For the smoothing parameter of 
the "medium-sized" policies has been chosen $\alpha=0.2$. The purpose of this choice is that the fluctuation of the premiums would be of a suitable magnitude from the policyholders point of view. On the basis of the insurer's statistics, a parameter $\alpha^{i}$ has been associated with every class $C_{i}$ so that the gain caused by the truncation (5.4) would be approximately of equal size for the different classes. This has led to an increasing sequence of parameters $\alpha^{i}$, $0.1 \leq \alpha^{i} \leq 0.28, i=1, \ldots, l$. A new policy starts in the tariff system with the smoothing parameter $\alpha^{i}$ defined by its initial premium.

The changing of $\alpha$ is carried out on the basis of the payroll $V_{n}$ of the policyholder (cf. 5.1). Every fifth year the size of the policy, measured by the payroll proportioned to the wage-index, is checked, and if the size has changed essentially, the parameter $\alpha$ is changed correspondingly.

The changing procedure is in accordance with assumption (4.6) (with $2^{\circ}$ ). Note first that it follows from assumption (4.6) that condition (4.3) holds true even if $X_{k}$ in (4.3) is replaced by $X_{k-1}, X_{k-2}$ or $X_{k-3}$. As a consequence, Corollary 4.2 remains valid if $X_{n}^{*}$ (see 5.2) is substituted for $X_{n}$ in (4.7). Further, it is reasonable to assume that the risks $\eta_{n, k}$ associated with single wage monetary units $k$ are independent of the size of the total payrolls. In addition, the straightforward use of the tariff is restricted to the case where there is not a significant trend in $\left(X_{n}\right)$ and the risk structure is stable. Note also that assumption (4.6) allows mutual correlations of the variables $\eta_{n, k}$.

Accordingly, by Corollary $4.2\left(Z_{n}\right)$, and hence $\left(p_{n}\right)$, can be considered to represent a correct tariff level.

\section{ACK NOWLEDGEMENTS}

I am grateful to Professor Esa Nummelin and Mr. Harri Nyrhinen for their comments on this paper.

\section{REFERENCES}

[1] Bonsdorff, H. (1989) A comparison of the ordinary and a varying parameter exponential smoothing. J. Appl. Prob. 26, 784-792.

[2] Brown, R.G. (1963) Smoothing, Forecasting and Prediction of Discrete Time Series. Prentice Hall, Englewood Cliffs, N.J.

[3] Gerber, H.U. and Jones, D.A. (1975) Credibility formulas of the updating type. In: Credibility: Theory and Applications. (ed. KAHN, P.M.), pp. 89-105. Academic Press, New York.

[4] Kremer, E. (1982) Exponential smoothing and credibility theory. Insurance: Math. Econ. 1, 213-217.

[5] Rantala, J. (1984) An application of stochastic control theory to insurance business. Acta Universitatis Tamperensis, A, 164, Tampere.

[6] Simberg, H. (1964) Individuelle Prämienregelung, eine Art des "Experience Rating". Trans. 17th Internat. Congr. of Actuaries, London Edinburgh 3, 650-659.

[7] SundT, B. (1988) Credibility estimators with geometric weights. Insurance: Math. Econ. 7, $113-122$.

\section{HeIKKI BonsdorfF}

Pohjola Insurance Company Ltd., Lapinmäentie 1, 00300 Helsinki, Finland. 